\section{Response to: 'Elephant in the room' by Hartung et al}

We read the letter to the editor 'Elephant in the room' by Hartung et al in response to our open-label pilot clinical trial report on the use of repository corticotropin injection (RCI) in refractory polymyositis (PM) and dermatomyositis (DM). ${ }^{12}$

First, the authors believe that the population studied may not be truly resistant given that only 3 of the 10 patients were treated with intravenous immunoglobulin (IVIg) before entering the trial. There are strong data to support that patients with myositis who have failed 40-60mg slow prednisolne taper and an average of 2.6 immunosuppressive drugs in addition to nearly $20 \mathrm{mg}$ of prednisone at study entry are considered. In the largest international multicentre randomised clinical trial done on PM and DM-the Rituximab in Myositis (RIM) trial, which included many international myositis experts, subjects failed an average of 3.1 immunosuppressive drugs in addition to at least $20 \mathrm{mg}$ of prednisone. ${ }^{3}$ Our pilot cohort is clearly consistent with RIM trial entry criteria in terms of subject refractoriness. Further, there is no clear consensus definition of 'refractory myositis' and, in general, patients failing high dose glucocorticoids plus one additional immunosuppressive agent in adequate doses for a reasonable period of time can be considered 'refractory'. Regarding IVIg, although we agree that IVIg is considered a reasonable immunomodulatory agent in patients with refractory DM (and perhaps PM), it is not a Food and Drug Administration (FDA)-approved therapy. Further, there are significant barriers for patients to receive IVIg including insurance approval, the significant expense of the drug and the onerous nature of a 2 to 5 -day long-duration intravenous infusion which is not practical for many working patients.

Second, the authors are questioning the safety and tolerability profile of the RCI in the study. We would like to point out that out of three serious adverse events (SAE), two were in the same patient (disseminated herpes zoster and avascular necrosis), and that patient was on mycophenolate, azathioprine and prednisone concomitantly at the time of the SAEs. We believe it was the combination immunosuppressive therapy that led to these unfortunate SAEs. Certainly, this is a lesson learnt for future clinical trials. Despite the SAEs and the patient being afforded the option of discontinuing study drug, this patient elected to continue the RCI (after temporary discontinuation) due to significant efficacy that she was experiencing for her severe refractory DM. Moreover, the frequency and severity of SAEs and adverse events (AE) in this open-label trial were consistent with other clinical trials in myositis where subjects received multiple concomitant immunosuppressive medications. The RIM trial had 67 SAEs and 308 AEs as well as infusion reactions (15\%) on 200 patients with myositis, and yet was considered safe and tolerable therapy for refractory disease.

Third, the authors comment about 'promotional' language as absolutely not justified. Our goal in performing this trial was to provide data from a prospective open-label study that went beyond the case reports that were previously published. We specifically mentioned that despite FDA approval, there are limited data on its clinical utility in myositis, and categorically stated that ours was a 'proof of concept' study and further studies are required to prove the merit of the drug. We provided detailed mechanistic plausibility and preliminary data for proposed steroid-dependent and independent hypotheses of RCI. ${ }^{4}$ Further, the blood sample repository that we concomitantly collected in the trial was done to allow for detailed mechanistic studies that would provide valuable data as to whether there was justification for a steroid-independent immunosuppressive effect for RCI. Moreover, we stated that despite the apparent favourable results of this pilot trial, the efficacy and safety of RCI need to be proven by a larger randomised clinical trial in myositis.

Fourth, we concur with the authors that RCI needs to be held by the same standards of efficacy and safety in prospective, double-blind, placebo controlled trials. However, our pilot clinical trial provided a much higher level of evidence than earlier 'case reports', given that it was prospective with predetermined inclusion and exclusion criteria as well as the incorporation of validated outcome measures. Further, no change in concomitant therapies including physical therapy was allowed throughout the trial period. AEs were monitored and reported in a standardised fashion throughout the study using the NIH's Common Terminology Criteria for Adverse Events v4.0. The 'proof of concept' terminology refers to the scientific methodology for the trial with no relationship to earlier FDA 'approval' of RCI in the 1950s.

Fifth, there are various levels of evidence-based support for any therapeutic agent for any disease from case reports or retrospective studies, to prospective open-label trial, and finally to a randomised controlled trial. ${ }^{5}$ Each study collectively provides evidence in support or against a scientific hypothesis. Therefore, we fail to understand why the authors believe that a higher level of evidence in a clinical trial using rigorous methodology nullifies earlier evidence of efficacy in published case studies when the findings point towards the same direction. ${ }^{6-8}$

Finally, we strongly disagree that we failed to mention the 'elephant in the room', that is, cost of the drug. We, in fact, categorically stated that RCI is unlikely to be first-line therapy given the high cost of the drug. Moreover, we recommended that a cost benefit analysis should be done in the future to determine the role of RCI in the treatment algorithm of myositis. In a retrospective study, patients with DM/PM receiving RCI had a lower mean hospitalisation rate, emergency room visits or outpatient visits, as compared with propensity-matched patients with DM/ PM receiving IVIg, rituximab or a combination of the latter agents. ${ }^{9}$ Unfortunately, these investigators failed to compare the medication cost or differences in efficacy, which is necessary to do in such a comparison.

We agree with the authors' final conclusion that a cost benefit analysis of RCI should be considered before recommending this drug, which is generally true of any of the newer expensive therapies including biological therapies for various rheumatic diseases.

\section{Rohit Aggarwal, Chester V Oddis}

Division of Rheumatology and Clinical Immunology, Department of Medicine, University of Pittsburgh, Pittsburgh, Pennsylvania, USA

Correspondence to Dr Rohit Aggarwal, Division of Rheumatology and Clinical Immunology, Department of Medicine, University of Pittsburgh, Pittsburgh, PA 15260,USA; aggarwalr@upmc.edu

Handling editor Josef S Smolen

Contributors RA and CVO contributed to scientific content of the manuscript.

Funding This research received no specific grant from any funding agency in the public, commercial or not-for-profit sectors.

Competing interests RA and CVO received an honorarium from Mallinckrodt for an advisory board unrelated to this trial.

Provenance and peer review Commissioned; internally peer reviewed.

(c) Article author(s) (or their employer(s) unless otherwise stated in the text of the article) 2019. All rights reserved. No commercial use is permitted unless otherwise expressly granted.

Check for updates

To cite Aggarwal R, Oddis CV. Ann Rheum Dis 2019;78:e12 
Received 11 February 2018

Accepted 11 February 2018

Published Online First 22 February 2018

\section{(S) Linked}

http://dx.doi.org/10.1136/annrheumdis-2018-213008

Ann Rheum Dis 2019:78:e12. doi:10.1136/annrheumdis-2018-213020

\section{REFERENCES}

1 Hartung DM, Johnston K, Cohen DM, et al. Elephant in the room. Ann Rheum Dis 2019;78:e11.

2 Aggarwal R, Marder G, Koontz DC, et al. Efficacy and safety of adrenocorticotropic hormone gel in refractory dermatomyositis and polymyositis. Ann Rheum Dis 2018;77:720-7.
3 Oddis $\mathrm{CV}$, Reed AM, Aggarwal R, et al. Rituximab in the treatment of refractory adult and juvenile dermatomyositis and adult polymyositis: a randomized, placebo-phase trial. Arthritis Rheum 2013;65:314-24.

4 Montero-Melendez T. ACTH: The forgotten therapy. Semin Immunol 2015;27:216-26.

5 Burns PB, Rohrich RJ, Chung KC. The levels of evidence and their role in evidence-based medicine. Plast Reconstr Surg 2011;128:305-10.

6 Patel A, Seely G, Aggarwal R. Repository Corticotropin Injection for Treatment of Idiopathic Inflammatory Myopathies. Case Rep Rheumatol 2016;2016:1-4.

7 Levine T. Treating refractory dermatomyositis or polymyositis with adrenocorticotropic hormone gel: a retrospective case series. Drug Des Devel Ther 2012;6:133-9.

8 Zhou Y, Lower EE, Li H, et al. Sarcoidosis patient with lupus pernio and infliximabinduced myositis: Response to Acthar gel. Respir Med Case Rep 2016;17:5-7.

9 Knight T, Bond TC, Popelar B, et al. Medical resource utilization in dermatomyositis/ polymyositis patients treated with repository corticotropin injection, intravenous immunoglobulin, and/or rituximab. Clinicoecon Outcomes Res 2017:9:271-9 